\title{
Numerical Simulation of the Boundary Layer Flow Generated in Monterey Bay, California, by the 2010 Chilean Tsunami: Case Study
}

\author{
Makris, Athanasios ; R. Lacy, Jessica ; Fuhrman, David R.
}

Published in:

Journal of Waterway, Port, Coastal, and Ocean Engineering

Link to article, DOI:

10.1061/(ASCE)WW.1943-5460.0000673

Publication date:

2021

Document Version

Peer reviewed version

Link back to DTU Orbit

Citation $(A P A)$ :

Makris, A., R. Lacy, J., \& Fuhrman, D. R. (2021). Numerical Simulation of the Boundary Layer Flow Generated in Monterey Bay, California, by the 2010 Chilean Tsunami: Case Study. Journal of Waterway, Port, Coastal, and Ocean Engineering, 147(6), [05021012]. https://doi.org/10.1061/(ASCE)WW.1943-5460.0000673

\section{General rights}

Copyright and moral rights for the publications made accessible in the public portal are retained by the authors and/or other copyright owners and it is a condition of accessing publications that users recognise and abide by the legal requirements associated with these rights.

- Users may download and print one copy of any publication from the public portal for the purpose of private study or research.

- You may not further distribute the material or use it for any profit-making activity or commercial gain

- You may freely distribute the URL identifying the publication in the public portal 
Case study:

\title{
Numerical simulation of the boundary layer flow generated in Monterey Bay, California by the 2010 Chilean tsunami
}

\author{
Athanasios Makris ${ }^{1}$, Jessica R. Lacy ${ }^{2}$, and David R. Fuhrman ${ }^{3, *}$ \\ ${ }^{1}$ Master of Science Student, Technical University of Denmark, Department of Mechanical \\ Engineering, Nils Koppels Alle, Building 403, 2800 Kgs. Lyngby, Denmark \\ ${ }^{2}$ Research Oceanographer, U.S. Geological Survey, Pacific Coastal and Marine Science \\ Center, 2885 Mission St., Santa Cruz, California, USA, 95060 \\ ${ }^{3}$ Associate Professor, Technical University of Denmark, Department of Mechanical \\ Engineering, Nils Koppels Alle, Building 403, 2800 Kgs. Lyngby, Denmark \\ ${ }^{*}$ Corresponding author, email: drf@mek.dtu.dk
}

\begin{abstract}
This work presents a case study involving the numerical simulation of the unsteady boundary layer generated by the 2010 Chilean tsunami, as measured by field equipment in Monterey Bay, California, USA. A one-dimensional vertical (1DV) boundary layer model is utilized, solving Reynolds-averaged Navier-Stokes equations, coupled with two-equation $k$ - $\omega$ turbulence closure. Local effects of convective acceleration (converging-diverging effects) on the boundary layer due to the sloping bed are likewise approximated. Four cases are considered involving simulation of: (1) the long tsunami-induced boundary layer flow in isolation, in combination with either (2) convective acceleration effects or (3) energetic short wind waves, and finally (4) all effects combined. Reasonable agreement with field measurements is achieved, with model results similarly showing that the tsunami-induced boundary layer in this case only spans a fraction of the local water depth. Systematic comparison of the various cases likewise elucidates the likely significance of both local converging-diverging effects,
\end{abstract}


as well as interaction with the much shorter period wind waves, on the tsunami-generated boundary layer. In the latter case, analogy is drawn to well-known wave-current boundary layer interaction, with the boundary layer turbulence associated with the short wind waves inducing an effective wave roughness felt by the tsunami-induced flow, which effectively plays the role of the current. 


\section{INTRODUCTION}

Tsunamis most commonly occur in the aftermath of seismic events and are thus inherently unpredictable in nature. While many studies of tsunamis emphasize their run-up and inundation, the nature of their-induced boundary layer flows (essential e.g. for understanding their induced sediment transport and scour around coastal structures) has also been studied realistically in controlled experimental (Larsen et al. 2018; Tanaka et al. 1999) or numerical (Williams and Fuhrman 2016; Larsen et al. 2017; Larsen and Fuhrman 2019a; Larsen and Fuhrman 2019b; Tinh and Tanaka 2019; Tanaka et al. 2020) environments. Due to their infrequent and unpredictable occurrence, actual field data involving the detailed boundary layer flow structure induced by tsunamis is elusive, seemingly being limited to the measurements of Lacy et al. (2012), who were the first to measure detailed velocities within the boundary layer of a tsunami-induced flow.

Specifically, the event in question corresponds to the 2010 Chilean tsunami, which was caused by the $M_{w}=8.8$ (moment magnitude scale) 2010 Chilean earthquake on February 27 off the coast of Maule. The resulting tsunami devastated several coastal towns in southcentral Chile and damaged the port at Talcahuano. Wave heights of about $3 \mathrm{~m}$ were reported from Chilean Islands, while the height of the tsunami in the deeper water was measured to be approximately $25 \mathrm{~cm}$ (see e.g. DART station 32412), which according an expert from the Pacific Tsunami Warning Centre (Fryer 2015), is large enough to issue a warning in the areas around the Pacific ocean.

Approximately $14 \mathrm{hr}$ later, corresponding to propagation around nearly half the globe as shown in Fig. 1, on February 28, 2010 the tsunami arrived at Monterey Bay, California, USA. Fortunately, the United States Geological Survey (USGS) had at the time an upwardpointing acoustic Doppler current profiler (ADCP), and a downward-pointing pulse-coherent acoustic Doppler profiler (PCADP) deployed at a test site in the bay, and the latter was utilized to take the measurements within the local tsunami-induced boundary layer flow (Lacy et al. 2012). The sampling frequency was increased in anticipation of the tsunami's 
arrival, and the data obtained from the PCADP are likewise the basis for the forthcoming case study. Directed toward the bed, the equipment took measurements from approximately $0.05 \mathrm{mab}$ (meters above the bed) to $0.62 \mathrm{mab}$ with a spacing of $0.095 \mathrm{~m}$ vertically and at a frequency of $1 \mathrm{~Hz}$ for 25 minutes every half hour.

In the numerical case study which follows the one-dimensional vertical (1DV) model of Fuhrman et al. (2013) will be utilized to simulate and study the tsunami-induced boundary layer flow measured by Lacy et al. (2012). This model is based on the horizontal component of the incompressible Reynolds-averaged Navier-Stokes (RANS) equations in combination with the Wilcox (2006) $k$ - $\omega$ turbulence closure model. The present case study attempts to model this event, motivated to better understand both the tsunami-induced boundary layer flow itself, as well as the influence of various external or local factors. Specifically, the study aims to elucidate the importance of both convective acceleration (converging-diverging effects due to the local bed slope) as well as interaction with energetic short (wind) waves that were present during the captured event. An overview of the basic parameters used in this model, as presented by Lacy et al. (2012), are presented in Table 1.

\section{MODEL DESCRIPTION}

\section{Governing Equations}

a As mentioned above, the model employed in this investigation is that of Fuhrman et al. (2013), and it is likewise detailed in the recent book of Sumer and Fuhrman (2020) (see their Section 5.12). It solves a simplified version of the horizontal component of the incompressible Reynolds-averaged Navier-stokes (RANS) equations, and for the turbulence closure the twoequation $k$ - $\omega$ of Wilcox (2006) and Wilcox (2008) is used. The RANS equation is as follows:

$$
\frac{\partial \bar{u}}{\partial t}=-\frac{1}{\rho} \frac{\partial \bar{p}}{\partial x}+\frac{\partial}{\partial y}\left(\frac{\tau}{\rho}\right)-\underline{\left(\bar{u} \frac{\partial \bar{u}}{\partial x}+\bar{v} \frac{\partial \bar{u}}{\partial y}\right)}
$$

In the above $t$ is time, $(\bar{u}, \bar{v})$ are the (Reynolds averaged, as indicated by the overbar) velocities in the $(x, y)$ (horizontal and vertical) directions, $\bar{p}$ is pressure, and $\tau$ is the total 
(viscous plus turbulent) shear stress expressed as

$$
\frac{\tau}{\rho}=\left(\nu+\nu_{T}\right) \frac{\partial \bar{u}}{\partial y}
$$

where $\rho$ is the fluid density, with $\nu$ and $\nu_{T}$ respectively being the kinematic fluid and eddy viscosity. In what follows the $x$ axis can be more specifically interpreted as pointing in the cross shore direction (positive onshore).

Note that the underlined terms in (1) (and also in forthcoming equations (7), (8) and (14)) correspond to convective acceleration terms, which are included in the present study to potentially account for converging-diverging effects associated with a sloping sea bottom. These are approximated as follows. As described in detail by Fuhrman et al. (2013), the $x$ derivative in the convective terms can be locally approximated as:

$$
\frac{\partial}{\partial x}=\frac{S}{h}
$$

where $S$ is the local slope of the seabed and $h$ is the local water depth. Subsequently, the vertical velocity can be obtained from the local continuity equation:

$$
\frac{\partial \bar{u}}{\partial x}+\frac{\partial \bar{v}}{\partial y}=0
$$

After invoking (3), and integrating from the local seabed at $y=0$, the vertical velocity can be obtained through cumulative integration:

$$
\bar{v}=-\frac{S}{h} \int_{0}^{y} \bar{u} d y
$$

This method conveniently allows for the effects from $x$-variations (from the sloping bed) to be incorporated in the $1 \mathrm{DV}$ model, while still only requiring discretization in the vertical $y$-direction. 
1 To achieve turbulence closure, we will utilize the two-equation $k$ - $\omega$ model of Wilcox (2006). This model consists of a transport equation for the turbulent kinetic energy (per unit mass)

$$
k=\frac{1}{2}\left(\overline{u^{\prime 2}}+\overline{v^{\prime 2}}+\overline{w^{\prime 2}}\right)
$$

where the prime superscript denotes turbulent fluctuations ( $w^{\prime}$ being the fluctuation in the unresolved horizontal along-shore $z$ direction) and the overbar averaging, corresponding to:

$$
\frac{\partial k}{\partial t}=\nu_{T}\left(\frac{\partial \bar{u}}{\partial y} \frac{\partial \bar{u}}{\partial y}\right)-\beta^{*} k \omega+\frac{\partial}{\partial y}\left[\left(\nu+\sigma^{*} \frac{k}{\omega}\right) \frac{\partial k}{\partial y}\right]-\underline{\left(\bar{u} \frac{\partial k}{\partial x}+\bar{v} \frac{\partial k}{\partial y}\right)}
$$

as well as a similar transport equation for the specific dissipation rate $\omega$ :

$$
\frac{\partial \omega}{\partial t}=\alpha \frac{\omega}{k} \nu_{T}\left(\frac{\partial \bar{u}}{\partial y} \frac{\partial \bar{u}}{\partial y}\right)-\beta \omega^{2}+\frac{\partial}{\partial y}\left[\left(\nu+\sigma \frac{k}{\omega}\right) \frac{\partial \omega}{\partial y}\right]+\frac{\sigma_{d}}{\omega} \frac{\partial k}{\partial y} \frac{\partial \omega}{\partial y}-\underline{\left(\bar{u} \frac{\partial \omega}{\partial x}+\bar{v} \frac{\partial \omega}{\partial y}\right)}
$$

The eddy viscosity $\nu_{T}$ is defined by

$$
\nu_{T}=\frac{k}{\tilde{\omega}}, \quad \tilde{\omega}=\max \left\{\omega, C_{l i m} \frac{|\partial \bar{u} / \partial y|}{\sqrt{\beta^{*}}}\right\}
$$

where $C_{\text {lim }}=7 / 8$. In $(8)$

$$
\sigma_{d}=\mathscr{H}\left\{\frac{\partial k}{\partial y} \frac{\partial \omega}{\partial y}\right\} \sigma_{d o}
$$

where $\mathscr{H}\{\cdot\}$ is the Heaviside step function, taking a value of zero when the argument is negative, and a value of unity otherwise.

In the right hand side of (7) the first term represents the production of turbulent kinetic energy (the rate at which kinetic energy is transferred from the mean flow to the turbulence), the second term represents dissipation (the rate at which turbulent kinetic energy is converted into thermal internal energy) and the third term includes both molecular and turbulent diffusion. The default model closure coefficients suggested by Wilcox (2006) are utilized: $\alpha=13 / 25, \beta=\beta_{0} f_{\beta}, \beta_{0}=0.0708, \beta^{*}=9 / 100, \sigma=1 / 2, \sigma^{*}=3 / 5, \sigma_{d o}=1 / 8$. 
Note that for two-dimensional flows, as considered herein, $f_{\beta}=1$.

\section{Boundary conditions}

The 1DV model described above is subject to the following boundary conditions. The bottom boundary is considered a friction wall, with a no-slip boundary condition imposed:

$$
\bar{u}=\bar{v}=0, \quad y=0
$$

Furthermore, at the bottom boundary, a zero-gradient condition is imposed for $k$, the turbulent kinetic energy (per unit mass), $d k / d y=0$, which imposes a zero flux of turbulent kinetic energy through the sea bed. This condition is justified based on experimental measurements for steady flows on rough beds by Fuhrman et al. (2010) and Sumer et al. (2003). Fuhrman et al. (2010) also demonstrated that the zero-gradient condition allows for a natural development of the viscous sublayer near smooth walls while avoiding the creation of a fictitious viscous sublayer near rough walls. This is in contrast to simply forcing $k=0$ at the wall, which creates (and hence requires resolution of) a viscous sublayer in all circumstances. This zero-gradient condition has also previously been successfully employed in simulations of oscillatory wave boundary flows by Fuhrman et al. (2011), on both smooth and rough beds.

a The bottom boundary condition for the specific dissipation rate, $\omega$, is taken from Wilcox (2006):

$$
\omega=\frac{U_{f}^{2}}{\nu} S_{R}, \quad y=0
$$

where

$$
S_{R}= \begin{cases}\left(\frac{200}{k_{N}^{+}}\right)^{2}, & k_{N}^{+} \leq 5 \\ \frac{K_{r}}{k_{N}^{+}}+\left[\left(\frac{200}{k_{N}^{+}}\right)^{2}-\frac{K_{r}}{k_{N}^{+}}\right] e^{5-k_{N}^{+},} & k_{N}^{+}>5\end{cases}
$$

Here $k_{N}^{+}=k_{N} U_{f} / \nu$ is the roughness Reynolds number, $k_{N}=2.5 d$ is Nikuradse's equivalent sand grain roughness ( $d$ being the sediment grain diameter), and $U_{f}=\sqrt{\left|\tau_{b}\right| / \rho}$ is the 
instantaneous friction velocity, where $\tau_{b}=\tau(y=0)$ is the bed shear stress. The value $K_{r}=180$ (a calibration constant) is utilized in this model, which has been demonstrated by Fuhrman et al. (2010) to produce standard logarithmic velocity profile solutions for steady boundary layers when used in combination with the $\partial k / \partial y=0$ bottom boundary condition.

\section{Pressure gradient}

A specified horizontal pressure gradient is used to drive the flow within the model, based on a prescribed velocity $u_{0}$ at the top of the model domain, which will be based directly on measurements from Lacy et al. (2012). The pressure gradient has thus been implemented as follows:

$$
\frac{1}{\rho} \frac{\partial \bar{p}}{\partial x}=-\frac{\partial u_{0}}{\partial t}-\underline{u_{0} \frac{\partial u_{0}}{\partial x}}+\left.\frac{1}{\rho} \frac{\partial \tau}{\partial y}\right|_{y=y_{t o p}}
$$

This is seen to account for both unsteadiness and convective acceleration, in the standard way for free stream regions. Additionally, the third term in the right-hand side of (14) accounts for potential shear stress at the top-most point of the model domain $\left(y=y_{\text {top }}\right)$, which in the present case study may be non-zero. This addition is necessary, since the top of the model domain has been here chosen to coincide with the largest-elevation field measurements, where the shear stress may not be exactly zero. (The velocimeter was mounted at a fixed elevation above the bed, and it is therefore not, at any given moment, certain whether the top-most velocity measurement is inside or outside the boundary layer; We have found that without including this term there will be a slow drift in the top-most velocity away from what was measured.)

Finally it is worth mentioning that the model as described in Fuhrman et al. (2013) is also capable of accounting for so-called "conventional" boundary layer streaming, which involves additional approximations for the convective terms to account for spatial variations inherent within progressive (regular) waves. For progressive (regular) waves, the importance of these effects is well known to scale as $U_{0 m} / c \sim a k_{w}$, where $U_{0 m}$ is the characteristic free-stream 
velocity magnitude, $c$ is the wave celerity, $k_{w}=2 \pi / L$ is the wave number, $L$ is the wave length, and $a=U_{0 m} / \omega_{w}$, where $\omega_{w}=2 \pi / T$ is the angular frequency and $T$ the wave period. Such effects have been found to be negligible for primary tsunami wave scales considered here, however, due to the extremely long wave lengths (hence very small wave number $k_{w}$ and very large celerity c) associated with tsunamis. This is easily confirmed by substituting typical tsunami-scale values into the scaling parameter mentioned just above. These conventional streaming effects are thus not included in any of the simulations which follow, for the sake of simplicity. (This is also convenient, since neither the transient tsunami nor the irregular short wave field to be considered could be very well approximated by a regular wave assumption, hence inclusion of such additional effects would not be straight forward.)

\section{RESULTS}

\section{Description of cases}

All model results will be based on simulated flows driven by velocity signals measured at the top-most elevation by Lacy et al. (2012). The full data set is, as described by Lacy et al. (2012), organized in bursts of 25 min each, with 5 min between each burst. However, the main tsunami event transpired during the third burst, and this will therefore be the focus of the current analysis, and these measurements, corrected for ambiguity errors as described in Lacy and Sherwood (2004), are depicted in Fig. 2. In this figure, the grey line depicts the full measured signal i.e. including both the long tsunami-induced flow, as well as that induced by energetic short wind waves, which were also present. Additionally, the dark black line in Fig. 2 depicts the low-pass filtered signal from Lacy et al. (2012), where the flow associated with the short waves has been removed, leaving the tsunamiinduced velocity signal in isolation. Note that we have divided the tsunami-induced flow into regions of acceleration (depicted by the diamonds) and deceleration (represented by ' $\mathrm{x}$ ' markers), where each time instant (representing a $10 \mathrm{~s}$ average, each separated by approx. 2 min). is marked by a different color. The coloring at these instants will be maintained in the presentation of several forthcoming velocity profile comparisons. Note that the peak velocity 
(represented by both a diamond and an $\mathrm{x}$ on Fig. 2) is taken to represent both the (end of the) accelerating region and the (beginning of the) decelerating region, to serve as a clear and shared state of reference.

We will consider four separate cases, as summarized in Table 2. Each will be driven by one of the velocity signals depicted in Fig. 2, as stated previously. (The only modification is that the initial flow is ramped up from zero in order to match the prescribed initial conditions; This takes place long before the arrival of the main tsunami wave, and hence does not affect the results of present interest.) As seen in Table 2, Case 1 is the most basic, considering simulation of the transient boundary layer which develops beneath the long tsunami-induced flow in isolation (black line in Fig. 2). Cases 2 and 3, respectively, consider the addition of either convective terms (again, accounting for converging flow due to a sloping bed) or the short wave field (i.e. now using the grey line in Fig. 2). Finally, Case 4 combines all three effects. In all cases the local water depth is taken as $h=9 \mathrm{~m}$, and the bed slope as $S=0.012$, which has been estimated from the contour plot provided as Figure 1 from Lacy et al. (2012). 1. Nikuradse's equivalent sand grain roughness is taken as $k_{s}=2.5 d$. Note finally that Lacy et al. (2012) found that the tsunami and short wave field were approximately co-linear. Hence no attempt to account for effects associated with varying directionality between the tsunami and short waves has been made, though it is recognized that this is only an approximation due to likely directional spreading within the irregular short wave field.

It should finally be mentioned that switching on the convective terms (as in Cases 2 and 4) creates a slight drift in the velocities (an additional form of wave boundary layer streaming, see e.g. Chapter 6 of Sumer and Fuhrman (2020)). To ensure proper comparison with the field measurements the exact times when the model results are chosen for these cases is adjusted slightly, such that the velocity at (or near) the top matches the field data, enabling the most direct and clear comparison with the other measurement locations further below. 


\section{Case 1: Tsunami alone}

The most natural place to start the case-study of this tsunami event is by modeling the tsunami-induced flow in isolation, Case 1. Comparison between the measured (circles) and simulated (full lines) results, at the acceleration/deceleration instants marked in Fig. 2 , are depicted in Fig. 3. It is seen that even in this case, the simulated evolution of the tsunami-induced wave boundary layer resembles that measured, especially considering that the present model is uni-dimensional. Results during the acceleration stages are more accurate than in the deceleration stage. This is also shown quantitatively in Table 3, which summarizes the root-mean-square error in the simulated velocity profiles (relative to the measurements) during periods of acceleration and deceleration, at the peak, as well as in totality (i.e. the average of all depicted times). Consistent with the findings of Lacy et al. $(2012)$, it is seen that the boundary layer induced by the tsunami is largely contained within the modeled near-bed domain i.e. it spans only a fraction of the $9 \mathrm{~m}$ water depth. This finding is likewise consistent with the later general findings of Williams and Fuhrman (2016), and is important as it implies that quasi-steady friction formulations (e.g. widely used Manning or Chezy formulations which effectively assume the boundary layer spans the full flow depth) should be used with great care in tsunami simulations. It is emphasized that this simulated case accounts for neither the effects of the short waves nor the beach slope on the resultant boundary layer. As it is likely of the most practical interest, as well as for the sake of simplicity and to ease comparison, much of the discussion for the various cases that follow will focus on the effects of the profile at the flow peak. In the present case it is clear that the modeled boundary layer velocities near the bed at peak flow are signicantly underestimated.

\section{Case 2: Tsunami and convective terms (converging-diverging flow effects)}

As presented in the model description, the model is able to include second-order terms in the simulation, accounting for convective acceleration (converging-diverging flow) effects associated with a sloping bed. Fuhrman et al. (2009a) and Fuhrman et al. (2009b) have previously demonstrated that, for the idealized case involving sinusoidal free stream flows, 
the importance of these effects will scale according to the dimensionless parameter:

$$
\frac{S a}{h}
$$

where $a=U_{0 m} T /(2 \pi)$ is a length scale, corresponding to the amplitude of the free stream fluid particle motion. Invoking the characteristic values from Table 1, along with the local bottom slope $S=0.012$ (estimated from the contour plot provided as Figure 1 in Lacy et al. (2012)) yields: $S a / h \approx 0.07$. This is of the order $10 \%$, thus non-negligible and indicating the likely importance of convective acceleration due to beach slope in this context. For comparison, doing similarly but using the wind wave scales from Table 1 instead yields: $S a / h \approx 0.0008$ i.e. two orders of magnitude smaller (hence likely insignificant). As the wave- and tsunami-induced velocities are similar in magnitude, and the bottom slope itself is relatively mild, it is clearly the much larger period of the tsunami (estimated as 16 min in this case, compared to a characteristic $10 \mathrm{~s}$ for the wind waves) which significantly increases the likely importance of convective acceleration effects due to beach slope in the present application.

Based on the assessment above, it was therefore expected that the converging flow effects will have a noticeable effect on the tsunami-induced wave boundary layer. This is largely confirmed from the simulation of Case 2, which is otherwise identical to Case 1, but now with the convective-acceleration terms active. The resulting horizontal velocity profiles are presented in Fig. 4, including comparison with those measured in the same fashion as in Fig. 3. It is seen that, with these effects added, the model fits the measured velocity profile during acceleration and near peak flow better. This is in line with physical expectations, since it is well-known that near bed velocities are enhanced due to favorable pressure gradients in a converging flow. If we focus e.g. on the two measurement elevations nearest the bed at the peak flow, it is now seen that the computed near-bed boundary layer velocities at the peak are, in fact, slightly over-predicted in this case. The results during the deceleration stage 
are again much less accurate, however, and these differences are even more exaggerated than in Case 1. These differences in accuracy beneath the accelerating and decelerating flow can also be seen quantitatively in Table 3 .

\section{Case 3: Tsunami and wind waves}

In contrast to Cases 1 and 2, Case 3 will now consider the boundary layer flow driven by the full signal (grey line) in Fig. 2, containing measured velocities from both the tsunami as well as the short wind wave field. Convective acceleration effects are switched off for this case. Lacy et al. (2012) hypothesized that the presence of the short wave field would result in an increased "apparent roughness" on the much longer tsunami, analagous to that in classical wave-current interactions (Grant and Madsen 1979). The computed velocity profiles for this case are shown at selected instants against the measured profiles in Fig. 5 , similar to before. Comparing this case with Case 1, the increased "apparent roughness" effects are indeed directly confirmed, which retard the flow near the bed considerably. This is particularly apparent at and near the peak flow. Addition of the short waves likewise seems to improve the computed boundary layer flow throughout the event, especially during the deceleration stage. Indeed, unlike Cases 1 and 2, the simulated flow is now more accurate during deceleration than during acceleration, as is also quantified in Table 3 .

\section{Case 4: Tsunami and wind waves with convective terms}

Finally, Case 4 will combine the full velocity signal (both tsunami and short waves) with convective acceleration terms also active, thus representing the most complete physical simulation to be considered in the present case study. In Fig. 6 the velocity profiles are once again compared to those measured in the field, and it can be concluded that the most complete model does describe the evolution of the tsunami wave boundary layer in a satisfactory and balanced fashion. Indeed, investigation of Fig. 6reveals that this simulation gives the best prediction of the boundary layer structure at peak flow, while also maintaining reasonably similar error during both periods of acceleration and deceleration (Table 3). The predictions at other times are not perfect, but are still reasonable given the difficulty of 
simulating complicated field conditions, and likely importance of other factors not captured within a simple 1DV approach. Such factors are briefly discussed below.

\section{DISCUSSION}

Perhaps the most compelling result from the present case study is the direct demonstration and confirmation via computational fluid dynamics simulation that the wind waves impose an additional and clear apparent roughness on the transient boundary layer flow inducedy by the tsunami. This effect is well known from the theory of wave-current interactions, see Grant and Madsen (1979) as well as e.g. Fredsøe and Deigaard (1992). It was also hypothesized to have occurred in this case by Lacy et al. (2012). A simple waveplus-current analogy is also considered in a wave friction coefficient approach by Sumer and Fuhrman (2020). The present case study is believed to be the first to clearly and quantitatively demonstrate such an effect utilizing a computational fluid dynamics model of the turbulent boundary layer, driven directly by field measured tsunami-induced velocities. To illustrate the effects of the apparent roughness more clearly, selected velocity profiles from Case 4 (all effects included) and Case 2 (without short waves) are compared in Fig. 7. The measurements at the same instants are also included for completeness. The retarding effect of the short-wave-induced apparent roughness on the tsunami-induced flow is very clear, especially near the bed. Without accounting for this effect, if energetic wind waves are also locally present it is clear that tsunami models would likely over-predict near bed velocities. It can also be seen that neither model result predicts a velocity overshoot near the time of maximum free stream flow, though a slight overshoot is evident in the field measurements. Velocity overshoot commonly occurs e.g. in oscillatory wave boundary layers, as a consequence of phase leads in the near bed flow from a previous cycle. It does not, therefore, occur in the numerical simulations since the driving velocity signal induces an essentially a transient (rather than periodic) event.

Additionally, this case study also confirms the likely importance of convective-acceleration (converging flow) effects in the boundary layer flows induced by transient tsunamis due to 
a sloping bed, even when the local bed slope is seemingly rather mild, being $O(0.01)$ in the present case. This was hypothesized in this context by Fuhrman et al. (2009a), who argued that the experimental conditions considered by Sumer et al. (1993) were most likely relevant at tsunami scales, based on $a / h$ being $O(10)$, which feeds directly into the scaling parameter identified in (15) above. This effect is best elucidated by comparing the simulated flow from Case 4 (all effects included) with Case 3 (without convective acceleration) at selected instants, as done in Fig. 8. Here it is seen that the inclusion of convective acceleration increases the flow near the bed significantly. This again makes physical sense, since this will induce a favorable pressure gradient when the flow is positive i.e. in the converging direction.

We finally compare computed results for the friction velocity (hence bed shear stress) with those estimated by Lacy et al. (2012) in Fig. 9. Lacy et al. (2012) estimated the tsunamiinduced friction velocities via a fit to a logarithmic velocity profile based on velocities (lowpass filtered and averaged over $10 \mathrm{~s}$ to eliminate effects from wind waves) from the lowest three measurement locations, at times when the tsunami-induced boundary layer extended at least $30 \mathrm{~cm}$ form the bed. Conversely, the time variation of the computed bed shear stresses stem directly from the model i.e. equation (2) applied at $y=0$. Results from Cases 1, 2 and 4 are specifically compared in Fig. 9, such that effects associated with the various contributing factors may be clearly elucidated. As Cases 1 and 2 exclude the effects of the shorter wind waves, the model result may be compared directly with the estimates of Lacy et al. (2012). It is seen that while the inclusion of convective-acceleration effects (as in Case 2) increases the friction velocity slightly relative to Case 1, both model results are well beneath the field estimates. Since Case 4 has resolved the short wind waves directly, the model results for the friction velocity are not directly comparable to the estimates of Lacy et al. (2012), which again correspond to the tsunami-induced contribution. To enable comparison for Case 4 we have therefore low pass (Butterworth) filtered the computed bed shear stress $\left(\tau_{b}\right)$ results, to eliminate the higher-frequency short-wave contributions. These filtered results have then been converted to the friction velocities depicted as the solid black 
line in Fig. 9. Note that this follows a similar methodology as used by Lacy et al. (2012) to isolate the tsunami-induced velocity signal from the raw signal, leading to the results depicted in Fig. 2. While the peak in the friction velocity from the model occurs somewhat earlier than estimated from the field data, the magnitude and overall variation for this case are much closer to the field estimates than either Cases 1 or 2 . This finding is generally in line with our previous discussions, and quantitatively illustrates the additional flow resistance felt by the long tsunami due to the presence of the short wind wave field.

Obviously, none of the simulations considered in the present case study utilizing a 1DV approach have resulted in a perfect match with the field measurements. Such differences are likely due, at least in part, to the treatment of the tsunami- and wind-wave-induced flow as simply co-linear. This simplification neglects entirely any effects of directional spreading within the short wave field, which will inevitably give rise to a three-dimensional flow and turbulence field. Additional effects associated with any bed forms (e.g. increased roughness) that may have been present would also serve to complicate matters further. Despite such differences, the match achieved in the present case study can be considered as quite reasonable, especially given the well-known difficulty of reproducing complicated field conditions in numerical models. Comparisons with desired effects switched on and off, as done herein, have likewise proved useful in quantitatively elucidating their likely importance in such field conditions.

\section{CONCLUSIONS}

A numerical case study simulating the 2010 Chilean tsunami-induced boundary layer flow, as captured in field measurements at Monterey Bay, California, USA by Lacy et al. (2012), has been conducted. The study utilizes a one-dimensional vertical model, based on the horizontal component of the Reynolds-averaged Navier-Stokes (RANS) equation, coupled with $k$ - $\omega$ turbulence closure, with flow driven by pressure gradients derived directly from the measured velocities. Four cases have been considered involving the boundary layer induced by: (1) the tsunami in isolation, (2) additional inclusion of convective acceleration effects 
due to a sloping bed, (3) the tsunami in the presence of the short wind wave field, and (4) all effects combined simultaneously. While none of the results can be considered as perfect, likely due at least in part due to limitations with a one-dimensional vertical approach compared with full three-dimensional field conditions, the boundary layer flow is reasonably captured by the simulations. In particular, the simulation including all effects combined captures the peak flow most accurately, while also maintaining similar errors during regions of both flow acceleration and deceleration. Comparison of the various cases clearly demonstrates the likely importance of both convective acceleration (due to a local bed slope) as well as the presence of a "wave-induced roughness" effect similar to those in wave-plus-current flows, where the developing boundary layer under tsunami effectively plays the role of the current. The latter finding largely confirms that speculated by Lacy et al. (2012). Hence, both of these effects should be accounted for in practical tsunami simulations, especially those where the detailed boundary layer flow near the bed is of interest, e.g. studies of scour or sediment movement.

\section{DATA AVAILABILITY}

The near-bed velocity measurements in Monterey Bay during the arrival of the 2010 Chilean tsunami are available at the U.S. Geological Survey data release: https://doi. org/10.5066/P9T90P01 (Ferreira et al. 2020). 


\section{REFERENCES}

Ferreira, J. C. T., Lacy, J. R., and Hatcher, G. (2020). "Near-bed velocity measurements in Monterey Bay during arrival of the 2010 Chile Tsunami: U.S. Geological Survey data release, https://doi.org/10.5066/P9T90PO1.

Fredsøe, J. and Deigaard, R. (1992). Mechanics of Coastal Sediment Transport. World Scientific.

Fryer, G. (2015). "Tsunami expert on waves that followed Chile earthquake." AP Archive, <https://youtu.be/YsgHrCUXqiU?t=42s>.

Fuhrman, D. R., Dixen, M., and Jacobsen, N. G. (2010). "Physically-consistent wall boundary conditions for the k- $\omega$ turbulence model." J. Hydraul. Res., 48, 793-800.

Fuhrman, D. R., Fredsøe, J., and Sumer, B. M. (2009a). "Bed slope effects on turbulent wave boundary layers: 1 . Model validation and quantification of rough-turbulent results." J. Geophys. Res. Oceans, 114, C03024.

Fuhrman, D. R., Fredsøe, J., and Sumer, B. M. (2009b). "Bed slope effects on turbulent wave boundary layers: 2. Comparison with skewness, asymmetry, and other effects." J. Geophys. Res. Oceans, 114, C03025.

Fuhrman, D. R., Schløer, S., and Sterner, J. (2013). "RANS-based simulation of turbulent wave boundary layer and sheet-flow sediment transport processes." Coast. Eng., 73, 151166.

Fuhrman, D. R., Sumer, B. M., and Fredsøe, J. (2011). "Roughness-induced streaming in turbulent wave boundary layers." J. Geophys. Res. Oceans, 116, C10002.

Grant, W. D. and Madsen, O. S. (1979). "Combined wave and current interaction with a rough bottom." J. Geophys. Res., 84, 1797-1808.

Lacy, J. R., Rubin, D. M., and Buscombe, D. (2012). "Currents, drag, and sediment transport induced by a tsunami." J. Geophys. Res. Oceans, 117, C09028.

Lacy, J. R. and Sherwood, C. R. (2004). "Accuracy of a pulse-coherent acoustic doppler profiler in a wave-dominated flow." J. Atmospher. Oceanic Technol., 21(9), 1448-1461. 
Larsen, B. E., Arbøll, L. K., Frigaard, S., Carstensen, S., and Fuhrman, D. R. (2018). "Experimental study of tsunami-induced scour around a monopile foundation." Coast. Eng., 138, 9-21.

Larsen, B. E. and Fuhrman, D. R. (2019a). "Full-scale CFD simulation of tsunamis. Part 1: Model validation and run-up." Coast. Eng., 151, 22-41.

Larsen, B. E. and Fuhrman, D. R. (2019b). "Full-scale CFD simulation of tsunamis. Part 2: Boundary layers and bed shear stresses." Coast. Eng., 151, 42-57.

Larsen, B. E., Fuhrman, D. R., Baykal, C., and Sumer, B. M. (2017). "Tsunami-induced scour around monopile foundations." Coast. Eng., 129, 36-49.

Sumer, B. M., Chua, L. H. C., Cheng, N.-S., and Fredsøe, J. (2003). "Influence of turbulence on bed load sediment transport." J. Hydraul. Eng., 129, 585-596.

Sumer, B. M. and Fuhrman, D. R. (2020). Turbulence in Coastal and Civil Engineering. World Scientific.

Sumer, B. M., Laursen, T. S., and Fredsøe, J. (1993). "Wave boundary-layers in a convergent tunnel." Coast. Eng., 20, 317-342.

Tanaka, H., Sana, A., Kawamura, I., and Yamaji, H. (1999). "Depth-limited oscillatory boundary layers on a rough bottom." Coast. Eng. J., 41, 85-105.

Tanaka, H., Tinh, N. X., and Sana, A. (2020). "Transitional behavior of a flow regime in shoaling tsunami boundary layers." J. Marine Sci. Eng., 8, 700.

Tinh, N. X. and Tanaka, H. (2019). "Study on boundary layer development and bottom shear stress beneath a tsunami." Coast. Eng. J., 61, 574-589.

Wilcox, D. C. (2006). Turbulence Modeling for CFD. DCW Industries.

Wilcox, D. C. (2008). "Formulation of the $k-\omega$ turbulence model revisited." AIAA J., 46, $2823-2838$.

Williams, I. A. and Fuhrman, D. R. (2016). "Numerical simulation of tsunami-scale wave boundary layers." Coast. Eng., 110, 17-31. 


\section{List of Tables}

․ 1 Characteristic parameters for the observed tsunami event, as reported in Lacy et al. (2012). . . . . . . . . . . . . . . . . . . . 21

$2 \quad$ The four distinct cases that are the basis for all the results . . . . . . . . . . 22

a 3 Tabulation of the model root-mean-square error (RMSE) for each of the four

tsunami wave boundary layer simulation cases during acceleration, at the

peak, during deceleration. The total RMSE is also shown, corresponding to the mean of all depicted time levels. . . . . . . . . . . . . . . . 23 
TABLE 1. Characteristic parameters for the observed tsunami event, as reported in Lacy et al. (2012).

\begin{tabular}{clll}
\hline \hline Parameter & & Symbol & Value \\
\hline \multirow{3}{*}{ Tsunami } & Maximum depth averaged velocity & $U_{m, T}$ & $0.36 \mathrm{~m} / \mathrm{s}$ \\
& Period & $T_{T}$ & $16 \mathrm{~min}=960 \mathrm{~s}$ \\
& Maximum wave height & $H_{T}$ & $1.12 \mathrm{~m}$ \\
& Reynolds number & $R e_{T}=T_{T} U_{m, T}^{2} /(2 \pi \nu)$ & $2.0 \times 10^{7}$ \\
\hline \multirow{3}{*}{ Wind waves } & Representative orbital velocity & $U_{m, w}$ & $0.4 \mathrm{~m} / \mathrm{s}$ \\
& Period & $T_{w}$ & $10 \mathrm{~s}$ \\
& Significant wave height & $H_{s}$ & $1.3 \mathrm{~m}$ \\
& Reynolds number & $R e_{w}=T_{w} U_{m, w}^{2} /(2 \pi \nu)$ & $2.5 \times 10^{5}$ \\
\hline \hline & Water depth & $h$ & $9 \mathrm{~m}$ \\
& Mean sediment grain size & $d$ & $0.315 \mathrm{~mm}$ \\
\hline
\end{tabular}


TABLE 2. The four distinct cases that are the basis for all the results

\begin{tabular}{cccc}
\hline \hline Case & Tsunami & Convective terms & Short waves \\
\hline 1 & $\times$ & & \\
2 & $\times$ & $\times$ & \\
3 & $\times$ & & $\times$ \\
4 & $\times$ & $\times$ & $\times$ \\
\hline \hline
\end{tabular}


TABLE 3. Tabulation of the model root-mean-square error (RMSE) for each of the four tsunami wave boundary layer simulation cases during acceleration, at the peak, during deceleration. The total RMSE is also shown, corresponding to the mean of all depicted time levels.

\begin{tabular}{lrcccc}
\hline \hline \multicolumn{1}{c}{} & & \multicolumn{4}{c}{$\operatorname{RMSE}(\mathrm{m} / \mathrm{s})$} \\
\hline Case & Figure & Acceleration & Peak & Deceleration & Total \\
\hline 1 & 3 & 0.0099 & 0.0119 & 0.0183 & 0.0139 \\
2 & $\overline{4}$ & 0.0068 & 0.0087 & 0.0248 & 0.0151 \\
3 & $\mathbf{5}$ & 0.0213 & 0.0211 & 0.0121 & 0.0171 \\
4 & $\mathbf{6}$ & 0.0133 & 0.0056 & 0.0155 & 0.0136 \\
\hline \hline
\end{tabular}




\section{List of Figures}

व 1 A view of the more than $9000 \mathrm{~km}$ the tsunami covered from the earthquake epicenter to the site in Monterey Bay just south of the wharf . . . . . . . . . 25

a 2 A plot showing the field data of the full flow, the tsunami filtered from it, and

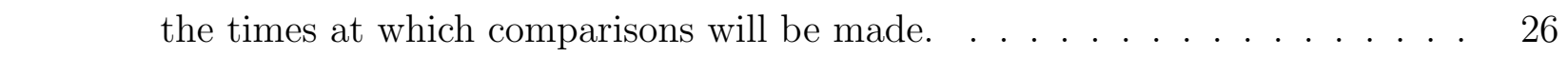

$3 \quad$ Results of the tsunami simulation (Case 1) compared to the field data. . . . 27

a 4 Results of the tsunami simulation including converging-diverging effects (Case 2) compared to the field data. . . . . . . . . . . . . . . . . . . 28

․ 5 Results of the tsunami and wind waves simulation (Case 3) compared to the field data. . . . . . . . . . . . . . . . . . . . . . . . 29

a 6 Results of the tsunami and wind waves simulation including converging-diverging effects (Case 4) compared to the field data . . . . . . . . . . . . . . . . . . . 30

व $\quad$ The effect of the wind waves on the evolution of the boundary layer compared to only modeling the tsunami. The converging diverging effects are included in the simulation. . . . . . . . . . . . . . . . . . . . . . . . . 31

a 8 The effect of the bed slope and therefore the converging-diverging effect on the evolution of the boundary layer. Both simulations include tsunami and wind waves. . . . . . . . . . . . . . . . . . . . . . . . . . . . . . . 32

a 9 Comparison of the computed tsunami-induced friction velocities (Cases 1, 2 a and 4) with the estimated field values of Lacy et al. (2012). To isolate the t. pass Butterworth filtering of the computed bed shear stress. . . . . . . . . . 33 


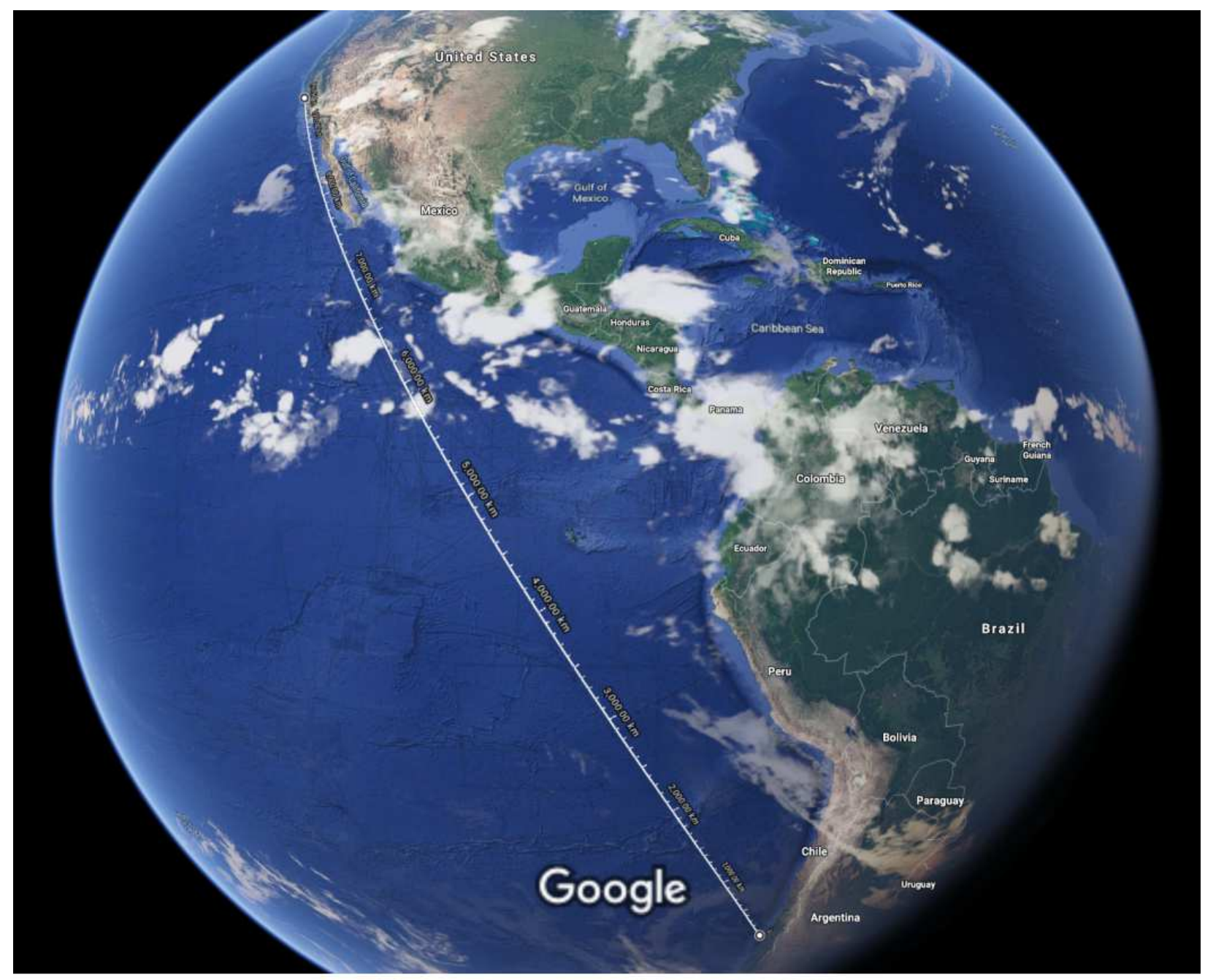

Imagery (C2021 Data SIO, NOAA, U.S. Navy, NGA, GEBCO, Landsat / Copernicus, IBCAO, U.S. Geological Survey, PGC/NASA, Imagery @2021 TerraMetrics, Map data @2021 INEGI

FIG. 1. A view of the more than $9000 \mathrm{~km}$ the tsunami covered from the earthquake epicenter to the site in Monterey Bay just south of the wharf. Figure created with Google maps. 


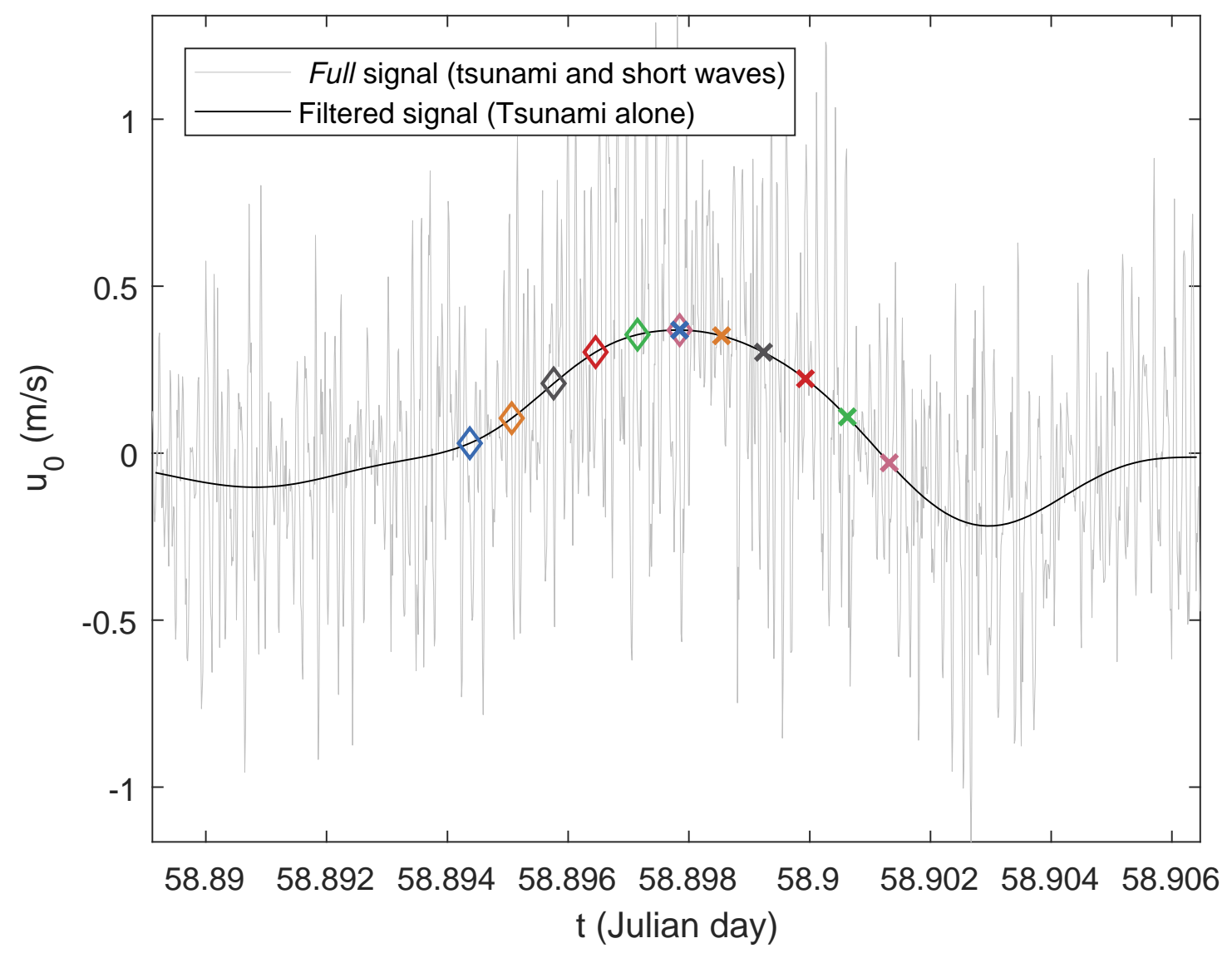

FIG. 2. A plot showing the field data of the full flow, the tsunami filtered from it, and the times at which comparisons will be made. 

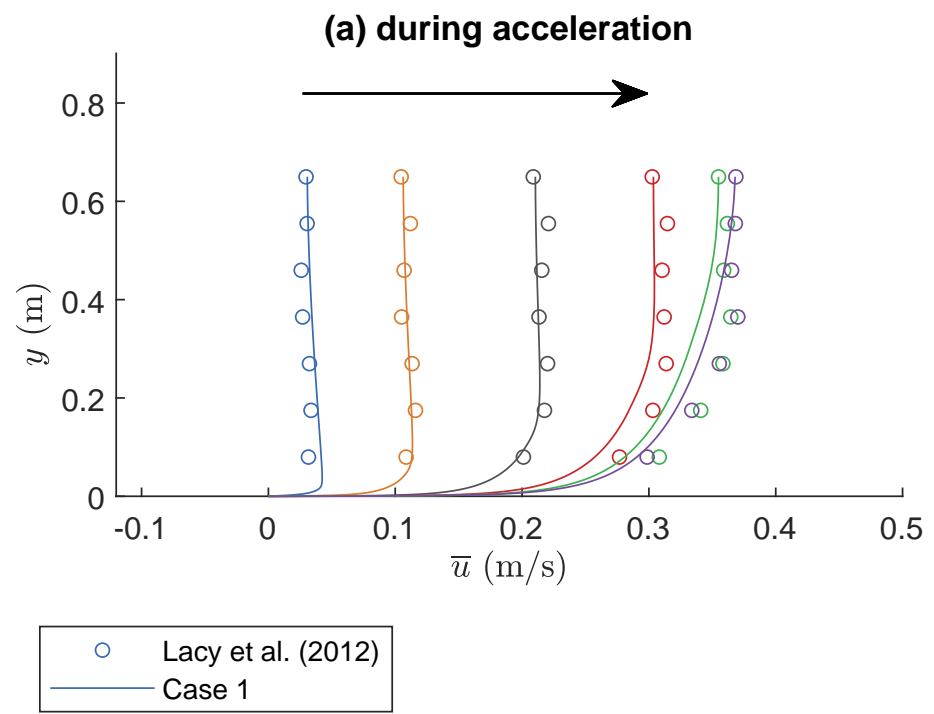

(b) during deceleration

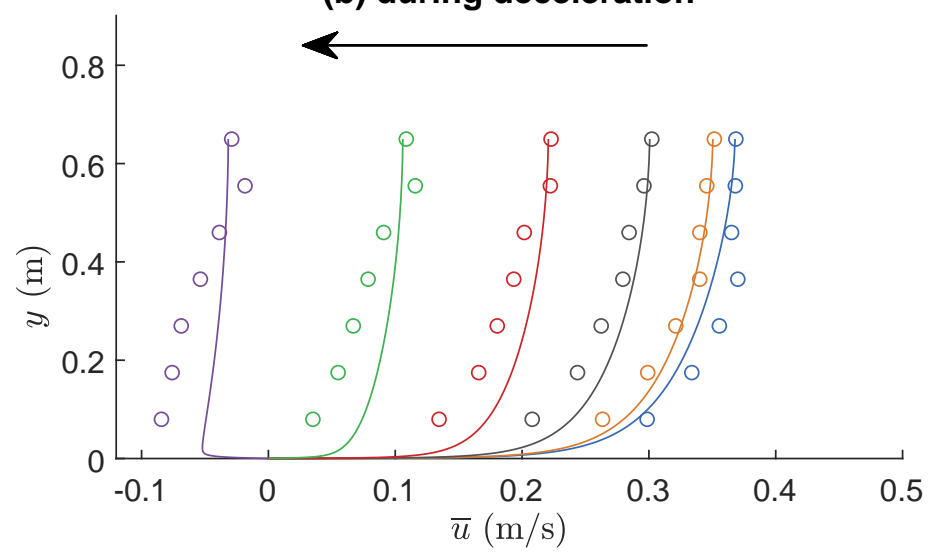

FIG. 3. Results of the tsunami simulation (Case 1) compared to the field data. 

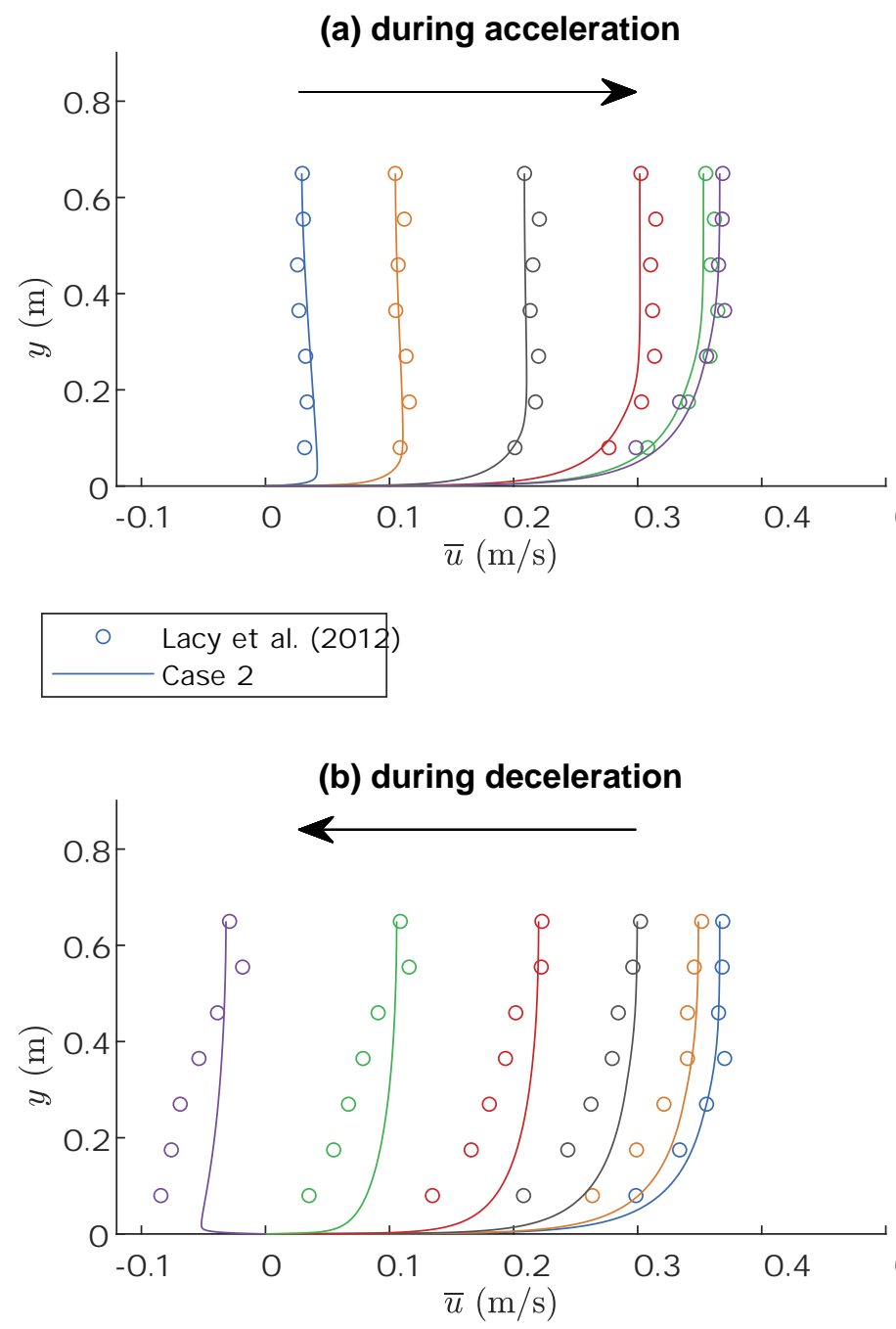

FIG. 4. Results of the tsunami simulation including converging-diverging effects (Case 2) compared to the field data. 
(a) during acceleration

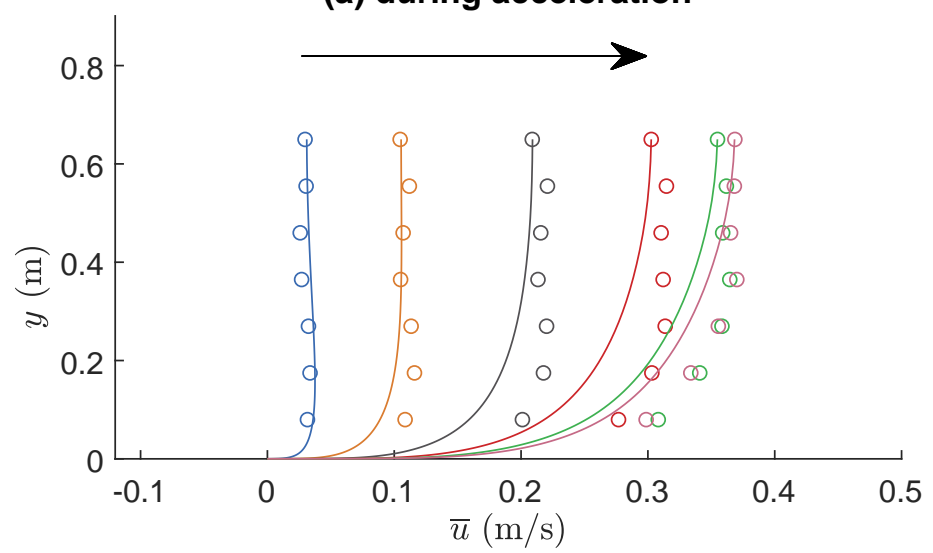

\begin{tabular}{|ll|}
\hline$\circ \quad$ Lacy et al. (2012) \\
Case 3
\end{tabular}

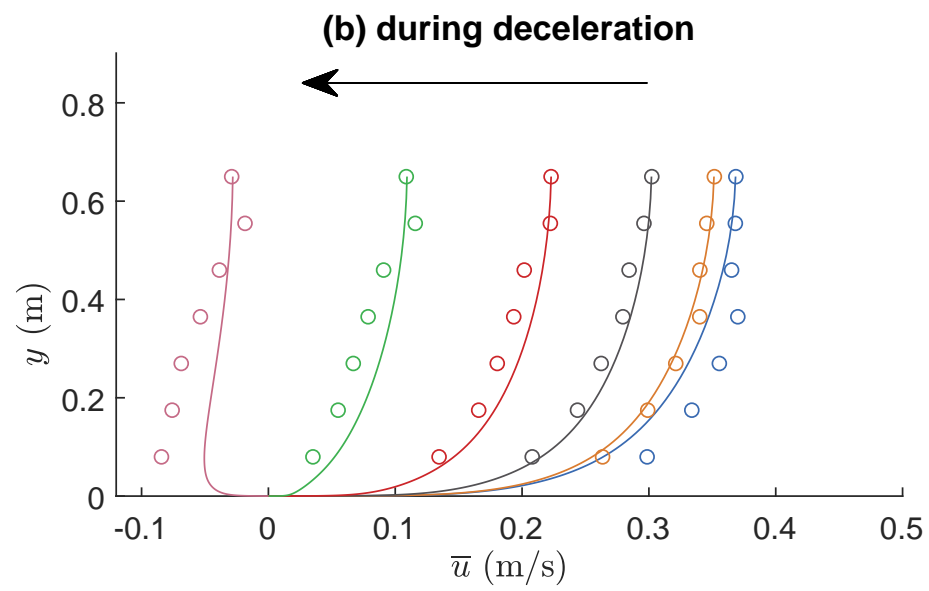

FIG. 5. Results of the tsunami and wind waves simulation (Case 3) compared to the field data. 

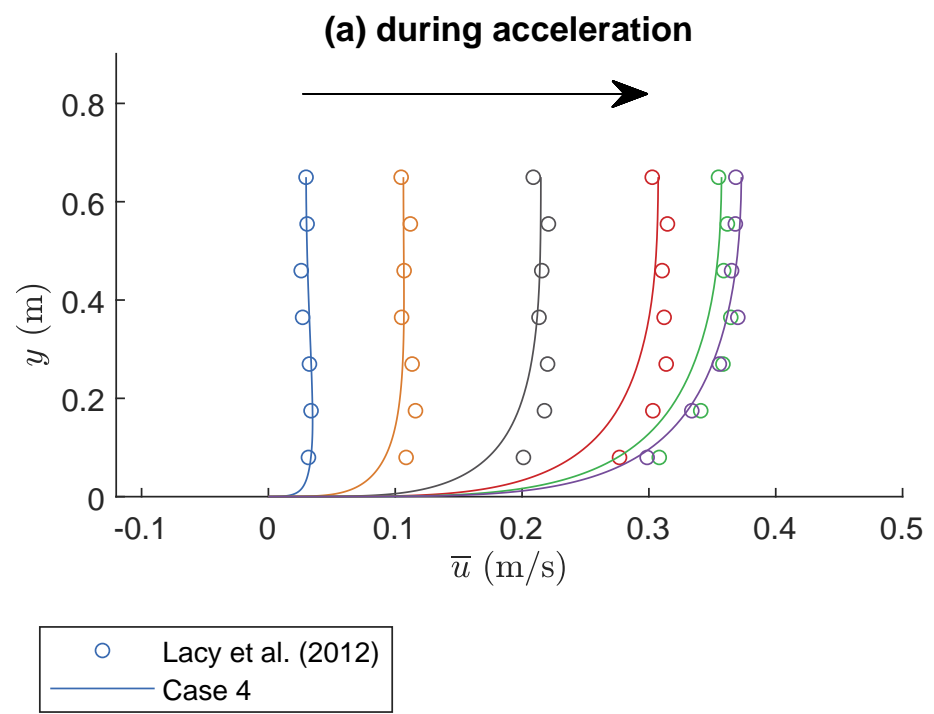

(b) during deceleration

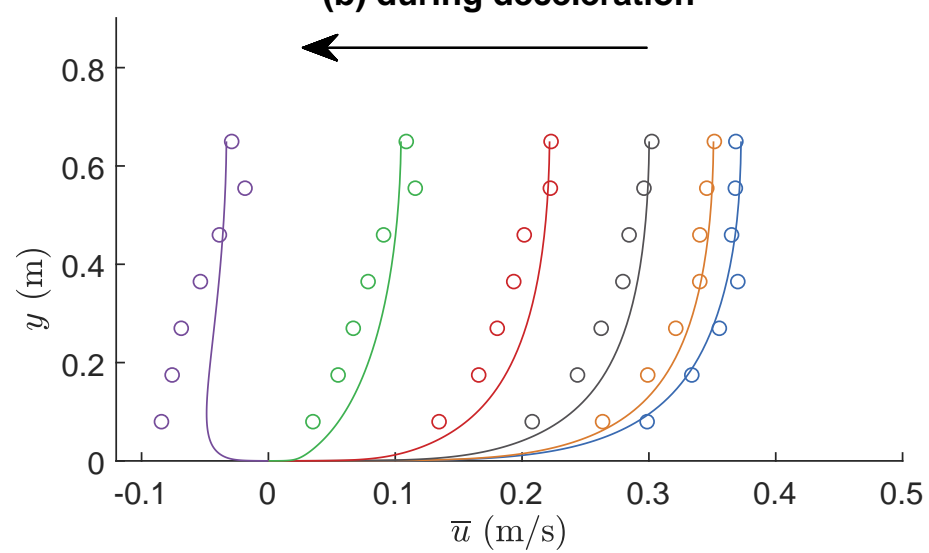

FIG. 6. Results of the tsunami and wind waves simulation including convergingdiverging effects (Case 4) compared to the field data. 
(a) during acceleration

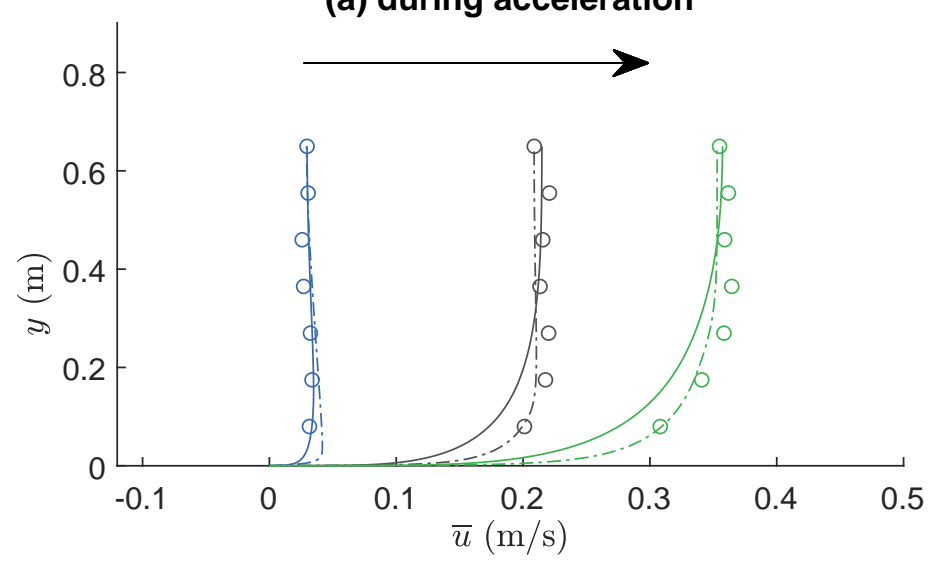

\begin{tabular}{|c|}
\hline Lacy et al. (2012) \\
Case 4 \\
$-\cdot-\cdot-\cdot-\cdot$ Case 2
\end{tabular}

(b) during deceleration

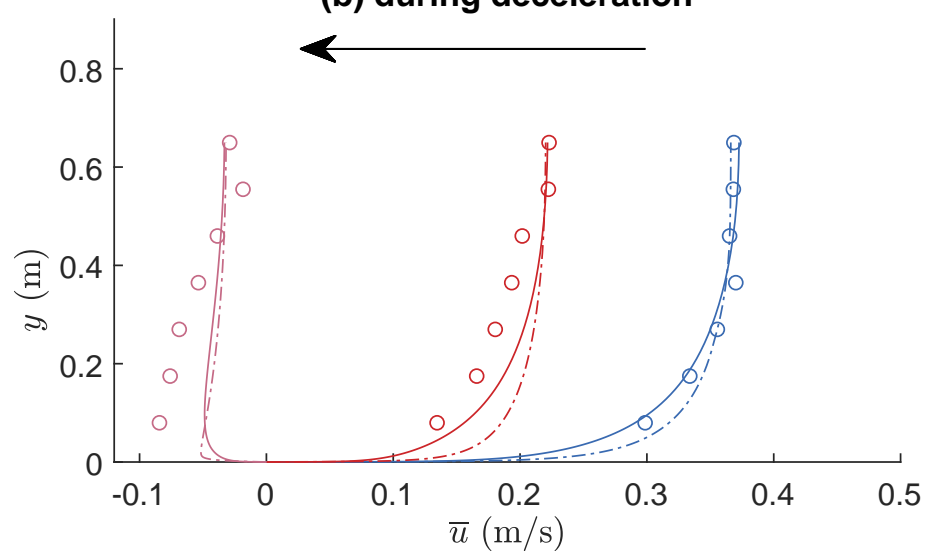

FIG. 7. The effect of the wind waves on the evolution of the boundary layer compared to only modeling the tsunami. The converging diverging effects are included in the simulation. 
(a) during acceleration

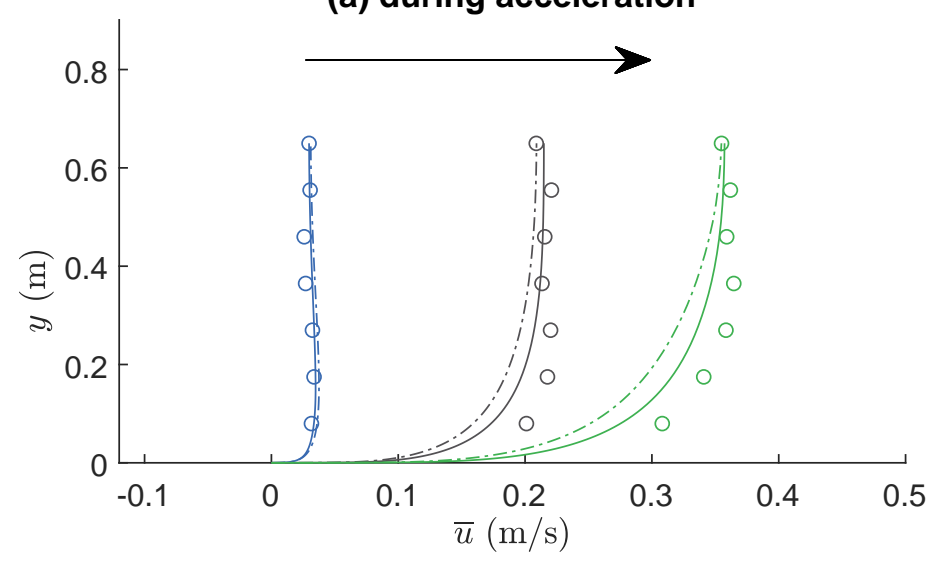

\begin{tabular}{|cl|}
\hline$\circ \quad$ & Lacy et al. (2012) \\
\hline & Case 4 \\
\hdashline$-\cdot$ & Case 3
\end{tabular}

(b) during deceleration

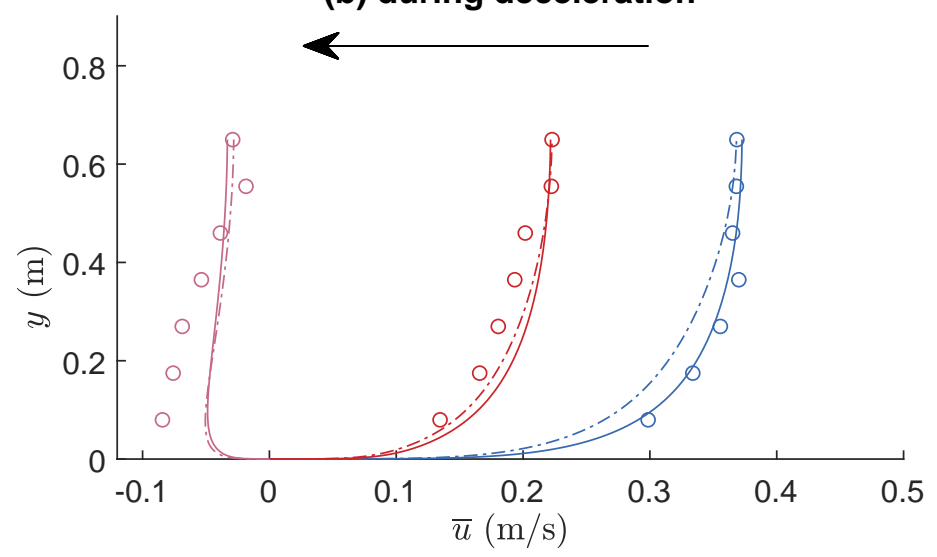

FIG. 8. The effect of the bed slope and therefore the converging-diverging effect on the evolution of the boundary layer. Both simulations include tsunami and wind waves. 


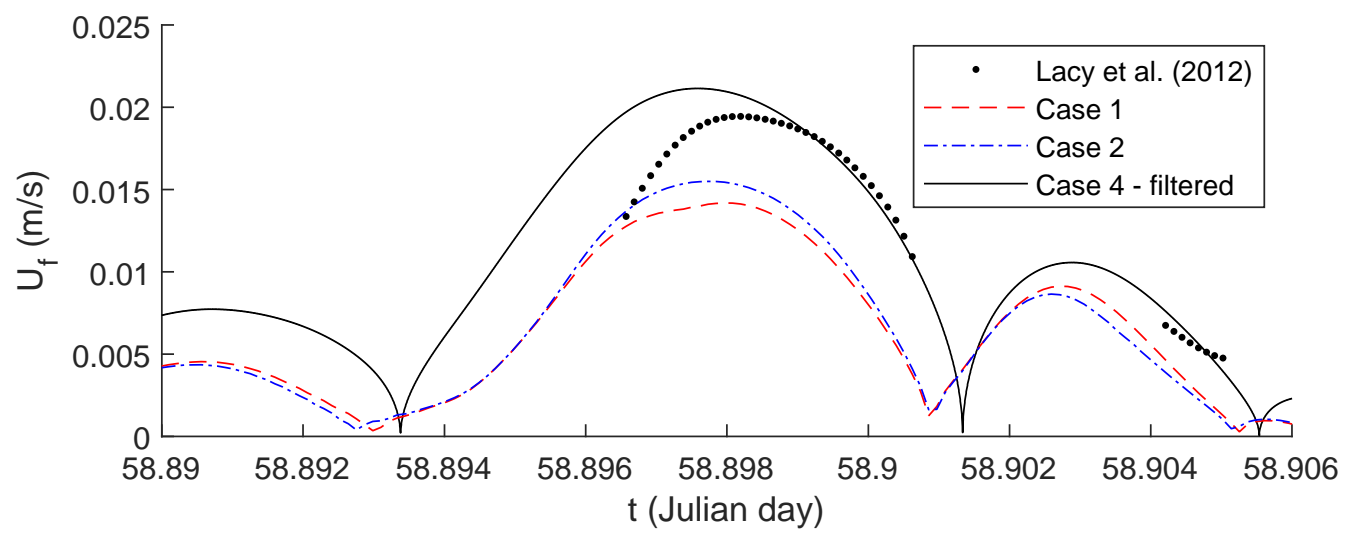

FIG. 9. Comparison of the computed tsunami-induced friction velocities (Cases 1, 2 and 4) with the estimated field values of Lacy et al. (2012). To isolate the tsunamiinduced contribution the Case 4 results have been obtained from low-pass Butterworth filtering of the computed bed shear stress. 